\title{
A Eficiência na Oferta de Alimentos por Pequenos Agricultores em Goiânia para Segurança Alimentar: uma análise pelo método Data Envelopment Analysis
}

\author{
Diogo Ferraz¹, Lie Yamanaka², Maico Roris Severino², Hélio Yochihiro Fuchigami² e \\ Daisy Aparecida do Nascimento Rebelatto ${ }^{3}$
}

O objetivo deste artigo é analisar a eficiência da oferta de alimentos com qualidade nutritiva e saudável para alunos de escolas públicas em Goiânia, a partir do oferecimento da agricultura familiar. A importância deste trabalho decorre da necessidade de alterar os hábitos alimentares de crianças e jovens, com base nos pressupostos da Segurança Alimentar. Além disso, comprovar empiricamente a eficiência dos alimentos oferecidos pelos pequenos agricultores é importante para encorajar projetos da Economia Solidária, estimulando a elevação da renda destes agentes. O método utilizado foi o Data Envelopment Analysis (DEA), por meio do modelo de Variable Return of Scale (VRS) orientado ao output. A escolha deste modelo se justifica devido a necessidade de levar em conta a escala de produção e a meta de aumentar o número de escolas atendidas pela agricultura familiar. Os resultados mostram que os alimentos oferecidos pelos pequenos produtores são eficientes em atender as escolas públicas de Goiânia, sobretudo, para os produtos agrícolas (Vegetais, Legumes e Frutas). Por outro lado, produtos elaborados ou semi-industrializados (alimentos enlatados, farinha e sêmola, laticínios) não demonstraram eficiência. Os resultados deste estudo sugerem que as escolas públicas podem receber alimentos frescos e saudáveis, por meio de pequenos agricultores da própria região. Isto implica no fortalecimento da agricultura familiar e na oferta de um cardápio mais nutritivo e saudável no ambiente escolar.

Palavras-chave: Agricultura familiar, Economia Solidária, Goiânia/GO-Brasil, Análise Envoltória de Dados (DEA).

\section{Efficiency in Food Supply by Small Farmers in Goiânia for Food Security: a Data Envelopment Analysis (DEA) approach}

The aim of this article is to analyze the efficiency of food supply with nutritious and healthy quality for students of public schools in Goiânia, from the offer of family agriculture. The importance of this work stems from the need to change the eating habits of children and young people, based on the assumptions of Food Security. In

\footnotetext{
1 Universidade de Hohenheim (Stuttgart/Alemanha) e Universidade de São Paulo (EESC/USP). Endereço para correspondência: Av. Trabalhador São-Carlense no 400, CEP 13566-590, São Carlos, SP, Brasil. Tel./Fax: (16) 3373 9425.E-mail. diogoferraz@usp.br

2 Universidade Federal de Goiás (UFG)

3 Universidade de São Paulo (EESC/USP).
} 
addition, empirically proving the efficiency of food offered by small farmers is important to encourage projects of the Solidarity Economy, stimulating the increase of the income of these agents. The method used was Data Envelopment Analysis (DEA), using the output-oriented Variable Return of Scale (VRS) model. The choice of this model is justified by the need to take into account the scale of production and the goal of increasing the number of schools served by family farming. The results show that the foods offered by the small producers are efficient in serving the public schools of Goiânia, especially for the agricultural products (Vegetables, Vegetables and Fruits). On the other hand, processed or semi-processed products (canned foods, flour and semolina, dairy products) have not shown efficiency. The results of this study suggest that public schools can receive fresh and healthy food from small farmers in the region. This implies strengthening family farming and providing a more nutritious and healthy menu in the school environment.

Keywords: Small Farmers in Brazil, Solidary Economy, Goiânia/GO-Brasil, Data Envelopment Analysis (DEA).

\section{INTRODUÇÃO E OBJETIVOS}

Nos países em desenvolvimento, a importância de formas alternativas de troca de mercadorias tem crescido. Nesse aspecto, muitas sociedades têm buscado formas mais inclusivas para gerar riqueza e bem-estar social, deixando de lado os modelos tradicionais do capitalismo[1,2,3].

Uma nova forma de organização da produção é a Economia Solidária[2], que busca a geração de trabalho e renda para pessoas excluídas do mercado de trabalho formal, encontrando nas coletividades (associações ou cooperativas) oportunidades para melhorar a qualidade de vida de sua família.

Note que a Economia Solidária está presente tanto nos espaços urbanos como nas áreas rurais. No caso das áreas rurais, há a presença da Economia Solidária na Agricultura Familiar, que busca por meio dos coletivos chegar aos mercados consumidores, especialmente, por meio dos programas de aquisição de alimentos governamentais, que seriam inacessíveis se atuassem individualmente[2,3].

No Brasil, vários agricultores familiares se tornaram fornecedores de produtos para a merenda escolar. No entanto, existem poucos estudos que analisam a eficiência desse processo para a Segurança Alimentar das crianças e jovens das escolas públicas. Assim, o objetivo deste artigo é analisar a eficiência da oferta de alimentos mais nutritivos para alunos de escolas públicas, cuja oferta ocorreu por meio de agricultores familiares.
Sabe-se que o Brasil tem superado os problemas da fome e da pobreza, utilizando políticas públicas que distribuem a renda de maneira justa $[1,4,5]$. A relação entre pobreza e insegurança alimentar foi analisada por modelos que estimam o consumo de determinado tipo de alimento. Essas descobertas relatam que a renda é um dos fatores mais importantes para favorecer uma dieta saudável. Qualquer tipo de insegurança alimentar tende a diminuir após a renda per capita do terceiro estrato familiar ( $\mathrm{R} \$ 40)$, ainda que algum tipo de alimento menos nutritivo aumente entre as classes sociais mais abastadas $[1,4,5,6,7,8,9]$.

Nesse cenário, a fome e a desnutrição não são mais o grande desafio. Estudos e políticas públicas têm se preocupado em analisar o tipo de alimento consumido por classes baixas e déficit educacional. Além disso, tem-se considerado que muitos produtos industrializados e de baixo custo, por exemplo, refrigerantes, pizza e salgadinhos, embora tenham conteúdo energético, são pobres em conteúdo nutricional. Por outro lado, produtos como frutas, legumes e verduras são considerados mais saudáveis e ricos em nutrientes ${ }^{[10,11,12]}$.

Este é um tema relevante, pois trata de dois aspectos sociais importantes para o desenvolvimento do Brasil. Primeiramente, analisa se os produtos oferecidos pela Agricultura Familiar são eficientes em relação ao preço médio e à quantidade ofertada de produtos semielaborados ou elaborados. Por outro lado, busca integrar a oferta desses produtos às pressões da Segurança Alimentar, para que alimentos 
mais saudáveis sejam oferecidos às crianças e jovens, em especial, àquelas de baixa renda alocadas nas escolas públicas, o que contribui para a mudança de hábitos alimentares no futuro.

Este artigo está dividido em quatro seções, além desta introdução. A segunda seção discute os conceitos de Economia Solidária e Segurança Alimentar. A terceira seção apresenta o método utilizado neste trabalho. A quarta seção discute os resultados encontrados e a quinta seção conclui.

\section{Economia Solidária, Pequenos Agricultores e Segurança Alimentar}

A economia solidária é um projeto de sociedade que busca uma nova forma de organização econômica como estratégia para enfrentar o desemprego e a exclusão social. A economia solidária rompe com as relações de produção capitalistas, introduzindo experiências populares baseadas em fundamentos de cooperação econômica e autogestão[10].

A cooperação econômica é fundamental para esse tipo de economia, que tem como princípio a propriedade coletiva e o direito à liberdade individual[2]. A autogestão, no entanto, corresponde à maneira pela qual esses empreendimentos são administrados. A gestão democrática é necessária para garantir a autogestão. Assim, a autogestão exige um esforço maior dos trabalhadores que, além das tarefas habituais, devem lidar com os problemas gerais da organização, e para isso é necessário que todos os parceiros tenham conhecimento do que acontece e possíveis alternativas para a tomada de decisões.

Essa nova forma econômica se projeta com os fundamentos da coletividade e solidariedade que são importantes para o ajuste das desigualdades sociais. No entanto, esta forma de organização econômica precisa enfrentar desafios, a fim manter a competitividade em relação às formas tradicionais de produção. Os principais desafios são: manutenção da consistência ideológica, contribuição técnico-científica, estruturação da comercialização e organização de políticas públicas ${ }^{[3]}$.

No que se refere à estruturação da comercialização e à organização de políticas públicas, há a oportunidade de fortalecer a Economia Solidária na Agricultura Familiar por meio do programa de compras governamentais denominado Programa Nacional de Alimentação Escolar (PNAE). Este programa é regido pela Lei no 11.947 de junho de 2009, que regula as diretrizes da alimentação escolar. Nestas diretrizes consta a preferência das escolas públicas pela aquisição de alimentos produzidos pela agricultura familiar. Além disso, a lei determina que pelo menos 30\% dos recursos financeiros repassados ao Fundo Nacional de Desenvolvimento da Educação (FNDE) pelo Fundo Nacional de Desenvolvimento da Educação (FNDE) sejam destinados ao agricultor familiar rural ou ao agricultor familiar.

Os agricultores familiares precisam decidir se participarão do programa, por meio de análise de viabilidade, além de produzir alimentos de qualidade com padrões nutricionais e sanitários de produção de alimentos.

Entretanto, os pequenos agricultores precisam estar preparados para mudanças nos hábitos alimentares, porque o padrão de consumo de alimentos no Brasil foi alterado ao longo dos anos. Essas mudanças vêm de uma série de fatores sociais e econômicos, que influenciam a preferência dos consumidores, como a urbanização, a faixa etária e a entrada da mulher no mercado de trabalho. Todos estes compõem um papel importante na composição da cesta de alimentos para a família ${ }^{[4]}$.

É importante observar os aspectos dos hábitos alimentares no Brasil. No final da década passada, o país estava prestes a atingir parte da meta mundial de Metas de Desenvolvimento do Milênio, estabelecida pela Organização das Nações Unidas (ONU), particularmente na redução da pobreza extrema e da desnutrição. Mais do que isso, a transição nutricional começou a mostrar que a maior parte da população brasileira está fora dos problemas relacionados à fome, no entanto, novos desafios surgiram. Entre eles, destaca-se a luta contra o sobrepeso e a obesidade, tornando o cenário mais complexo e garantindo a necessidade de analisar o consumo de alimentos nas classes brasileiras menos favorecidas ${ }^{[5]}$.

Além disso, questões sobre segurança alimentar passaram a fazer parte da agenda política do Brasil após a crise global de nutrição em 2007/2008. 
Esta questão tem atraído o interesse de pesquisadores, embora ainda careçam de estudos sobre a relação entre os pequenos agricultores e a segurança alimentar [12].

O primeiro passo para alcançar a segurança alimentar é o acesso à alimentação, embora, até onde se sabe, a fome não seja causada pela inexistência de comida, mas porque as pessoas não têm acesso aos alimentos. Sendo assim, a segurança alimentar existe quando os moradores de uma casa têm acesso regular e permanente a alimentos suficientes para suas necessidades nutricionais.

Estudos defendem que a segurança alimentar é um conceito multidimensional que apresenta a disponibilidade de alimentos (acesso físico à comida), a acessibilidade alimentar (acesso econômico aos alimentos), o uso de alimentos (absorção dos nutrientes pelo corpo) e a vulnerabilidade[12].

Para possibilitar essa transição, o programa governamental "Fome Zero" teve como objetivo a redução da fome, da desnutrição e da extrema pobreza no Brasil. Por outro lado, programas sociais como as transferências monetárias (Bolsa Família), o Beneficio da Prestação Contínua, as pensões rurais e a valorização do salário mínimo foram fatores muito importantes na redução da pobreza e insegurança alimentar das famílias mais pobres, particularmente desde 2001[1,6,7].

O Bolsa Família, por exemplo, contribuiu para o aumento da segurança alimentar e nutricional, pois $76 \%$ das transferências de renda são gastas em alimentos, garantindo uma dieta melhor para as famílias das classes mais baixas.

O aumento da renda é importante para a segurança alimentar, pois sabe-se que a elasticidade renda das despesas com a dieta aumenta com uma renda domiciliar per capita (RPC) maior, embora a elasticidade seja sempre menor do que 1 . Sendo assim, os recursos financeiros gastos com alimentos é igual a $60,2 \%$ na primeira classe de renda (RPC até R \$ 100), cai para 36,6\% na segunda classe (de $\mathrm{R} \$ 100$ para $\mathrm{R} \$$ 200) e continua caindo para apenas $5,2 \%$ na RPC classe que está acima de $\mathrm{R} \$ 4$ mil reais ${ }^{[8]}$. Segundo os autores, o arroz e o feijão têm elasticidade negativa, o que significa que um aumento proporcional na renda de todos os brasileiros não aumentará a demanda por esses produtos.
Embora a renda familiar seja a principal restrição à insegurança alimentar medida pela Escala Brasileira de Insegurança Alimentar (EBIA), Hoffmann[1,6,9] estudou os determinantes da probabilidade das famílias em consumir alguns tipos de alimentos.

Os dados da Pesquisa Nacional por Amostra de Domicílios (PNAD), em 2004, mostram que 34,9\% das residências ainda permaneciam com algum nível de insegurança alimentar[ [6]. Em 2009, apesar do número de residências analisadas ter aumentado para $12,6 \%$, o número daqueles em situação de insegurança moderada foi reduzido para $40,2 \%$ e o de insegurança severa caiu para $12,4 \%[9]$.

Quando se trata do tipo de consumo alimentar, há uma probabilidade maior no consumo de carne suína no Sul, enquanto, no Norte e Nordeste, ocorre o consumo de alimentos básicos (arroz, feijão, farinha de mandioca, leite em pó e margarina) ${ }^{[8]}$.

A farinha de mandioca é mais consumida no Norte e Nordeste do país. A taxa média de consumo de farinha de mandioca pode ser explicada pela localização, entre o urbano e o rural. O consumo desse bem é quatro vezes maior nas áreas rurais do que nas cidades, por exemplo[8].

Estima-se que há maior probabilidade de ocorrer insegurança alimentar nos locais urbanos após o controle do efeito da renda[6]. No entanto, as áreas rurais remotas tendem a ter residências mais pobres. A evolução desfavorável da segurança alimentar nos lares rurais de 2009 a 2013 pode ter ocorrido devido ao "rural" em 2013 ser uma das áreas mais restritas cujas residências são, em geral, relativamente pobres ${ }^{[1]}$.

Quando a probabilidade de ocorrência de insegurança alimentar é analisada pelo setor de ocupação por um membro da família de referência, há maior probabilidade para os trabalhadores do setor agrícola[$\left.{ }^{[}\right]$. A proporção de domicílios com extrema insegurança alimentar é quase três vezes maior entre aqueles em que a pessoa de referência é preta ou parda $(10 \%)$, do que entre aqueles cuja pessoa de referência é branca $(3,55 \%)^{[6]}$.

Observe que os negros e pardos têm maior probabilidade de adquirir açúcar, arroz, carne bovina de segunda e farinha de mandioca. Além disso, há uma 
probabilidade negativa de consumir carne bovina de primeira, banana, batatas, tomate, queijo e leite longa vida[4].

Esse resultado pode estar associado à elasticidade de renda de alguns produtos de alta qualidade ou relativamente caros, que são, dessa forma, respectivamente comprados em grande quantidade pelas famílias relativamente ricas e brancas. A flexibilidade da renda no consumo de carne bovina de primeira é maior que a carne bovina de segunda.

Assim, os resultados de Hoffmann $[1,6,8,9]$ relatam que as pessoas na pior faixa de renda têm agora mais probabilidade de consumir leite em pó, um tipo de alimento conhecido por sua maior elasticidade renda. Pelo menos, em alguns aspectos, o consumo de leite em pó pelos pobres pode ser justificado pela falta de geladeiras nas casas. Por isso, a presença de geladeiras aumenta a provável aquisição de leite longa vida ${ }^{[4]}$.

O histórico educacional da pessoa de referência também é uma mudança responsável pela ocorrência de segurança alimentar. Um ano adicional de escolarização diminui a insegurança alimentar grave $(-8 \%)$ e a insegurança alimentar leve, moderada ou severa $(-4,3 \%)[7$. Segundo o autor, o nível de escolaridade e a distribuição de renda transformam a escolha do consumidor por alimentos mais nutritivos.

Quando o chefe da família não possui nível de escolaridade elevado, a probabilidade de consumir açúcar, arroz, feijão, farinha de mandioca e carne bovina de segunda é maior, além disso, o aumento da educação, além da renda, aumenta a probabilidade de consumir alimentos orgânicos e leves/produtos dietéticos $^{[1]}$.

No que diz respeito aos familiares, a probabilidade de insegurança alimentar nas residências com uma ou duas pessoas é maior[]. Isso condiz com os dados da pesquisa internacional, em que o grande número de familiares ajuda a diluir os custos fixos de

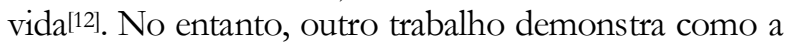
insegurança alimentar extrema nas residências com mais de sete pessoas também pode acontecer[ ${ }^{[]]}$.

Dados da PNAD (2013) mostram que a média de moradores por domicílio privado foi de 3,09 em geral, e 2,96 nos domicílios com segurança alimentar, 3,57 nos domićlios com insegurança leve, 3,46 nos domicílios com insegurança moderada e 3,43 nas residências particulares com insegurança alimentar extrema ${ }^{[1]}$. Entretanto, o número médio caiu de 4,14 em 2004 para 3,43 em 2013 nos lares com insegurança alimentar moderada ou extrema.

\section{MATERIAL E MÉTODOS}

A eficiência de um sistema pode ser medida a partir da divisão entre o valor corrente de um indicador de desempenho do sistema e o valor máximo que esse indicador atinge[13].

O método usado para determinar a eficiência de sistemas é chamado de Data Envelopment Analysis (DEA). O DEA é um método não paramétrico, que constrói de forma empírica uma fronteira linear por pares, para medir a eficiência produtiva de um conjunto de Unidades Tomadoras de Decisão ou Decision Making Unit (DMU)[14,15]. Uma DMU pode ser uma máquina, uma empresa, um país ou região, uma pessoa ou algum produto.

Neste estudo, as DMUs são os alimentos fornecidos pelos agricultores familiares, de forma a verificar quais produtos são mais vantajosos neste modelo de negócio (pequenos agricultores versus escolas públicas).

A partir da eficiência de cada DMU, um ranking de eficiência é gerado. A fronteira eficiente expressa o número máximo de outputs que podem ser produzidos por unidade de inputs, representando o limite de produção determinado pela tecnologia vigente[16].

Vários estudos analisam a eficiência dos sistemas de produção em diferentes aspectos, como o setor bancário[17], aeroportos ${ }^{[18]}$, setor industrial[19], cidades ${ }^{[20,21,22]}$, produtores rurais ${ }^{[23]}$ e países ${ }^{[24,25,26,27]}$. Embora o DEA tenha sido inicialmente desenvolvido para calcular a eficiência relativa em um processo produtivo, essa técnica tem sido aplicada para solucionar diversos problemas [24].

Uma das possibilidades é construir índices compostos (CI), que permitem avaliar o desempenho de diferentes situações. Este tipo de aplicação DEA, pode ser feito através de duas abordagens: (a) com um 
modelo contendo apenas outputs e um input igual a 1 (chamado modelo Benefit of the Benefit-BoD), ou (b) com modelos que contenham inputs e outputs que não expressem uma relação de produção (por exemplo, indicadores de custo-benefício)[24].

Neste sentido, neste artigo, entende-se por eficiência a capacidade dos pequenos agricultores em atender o maior número de escolas públicas de Goiânia, por meio de preços e quantidades adequadas para o consumo. Contudo, sabe-se que existem outros aspectos de eficiência que poderiam ser mensurados, por exemplo, a eficiência dos próprios agricultores, do processo produtivo, da terra, entre outros.

Para este trabalho, estudos de eficiência social podem ser utilizados como base teórica[24]. Vale ressaltar que muitos autores utilizaram o DEA para construir indicadores de desenvolvimento humano e bem-estar social[24,25,26].

Nosso trabalho difere dos demais porque utiliza grupos de alimentos como DMUs para medir a eficiência da segurança alimentar de alunos de escolas públicas atendidos por pequenos agricultores da região de Goiânia (GO/Brasil).

Existem diferentes modelos que podem ser usados para implementar o DEA. Esses modelos diferem de acordo com suas suposições. O tipo de retorno de escala designa os dois principais modelos DEA: CCR (Retorno Constante à Escala) e BCC (Retorno Variável à Escala).

A hipótese do modelo BCC considera que a variação dos outputs não é necessariamente equiproporcional aos imputs, sendo que na fronteira haverá três regiões: crescente, em que os outputs crescem proporcionalmente mais que os imputs, constante; e decrescente, onde os outputs crescem proporcionalmente menos que os inputs [13,16].

A Tabela 1 ilustra a formulação matemática dos principais modelos DEA.
Tabela 1. Principais modelos DEA

\begin{tabular}{|c|c|c|}
\hline & Orientado ao input & Orientado ao output \\
\hline C & 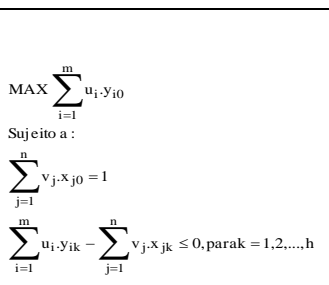 & 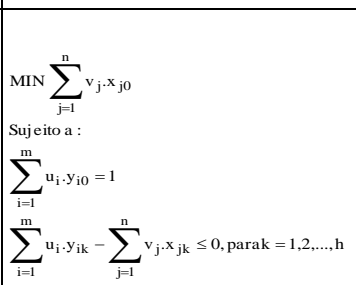 \\
\hline C & 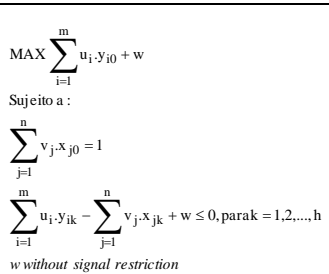 & 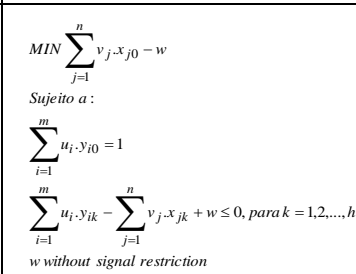 \\
\hline
\end{tabular}

Fonte: Mariano e Rebelatto (2014, p. 5).

Os dados utilizados nesta pesquisa são primários, coletados por dois grupos de pesquisa, com o apoio de um projeto financiado pelo British Council e pela Fundação de Pesquisa do Estado de Goiás (FAPEG).

Foram utilizados 90 tipos de alimentos (DMUs), categorizados de acordo com a Tabela de Composição Nutricional do Consumo Alimentar no Brasil da Pesquisa de Orçamentos Familiares (POF), que é a maior pesquisa de renda familiar do país, publicada pelo Instituto Brasileiro de Geografia Estatística (IBGE).

As categorias de alimentos analisadas são: Cereais e leguminosas, Laticínios, Açúcar e confeitaria, Produtos de panificação, Cames processadas, Óleos e gorduras, Aves e ovos, Açúcar e produtos de confeitaria, Diversos.

As variáveis escolhidas para compor a análise de eficiência foram: quantidade total de alimentos ofertados (imput), preço médio dos alimentos ofertados (input), preço total dos alimentos fornecidos (input). Como output, foi utilizado o número de escolas públicas atendidas. Sendo assim, a eficiência neste trabalho tem como parâmetro o preço e a quantidade dos produtos ofertados. O modelo DEA-BCC foi orientado ao output, pois deseja-se maximizar o output (número de escolas atendidas) sem reduzir os inputs 
(preço e quantidade). O software utilizado para essa análise de eficiência foi o MATLAB.

Para validar as variáveis, foi mensurada uma matriz de correlação de Pearson com significância estatística. $\mathrm{O}$ software utilizado para esta análise foi o STATA 13.0.

\section{RESULTADOS E DISCUSSÃO}

A matriz de correlação de Pearson demonstrou que os inputs e o output têm significância estatística e estão correlacionados. Por exemplo, a correlação entre as escolas atendidas e a quantidade de produtos ofertados foi de 0,6931, com nível de significância de $1 \%$. O preço dos produtos e as escolas atendidas apresentaram o coeficiente esperado pela literatura econômica $(-0,7452)$, considerando que quando o preço aumenta a quantidade demandada diminui. Essa correlação também mostrou um nível de significância de 1\%.

Justifica-se a utilização da matriz de correlação de Pearson, pois o objetivo da análise estatística é apenas comprovar a correlação entre os inputs e o output, o que permite a análise de eficiência por meio do DEA.

O modelo DEA-BCC orientado para o output avaliou a eficiência no processo de oferta de alimentos para escolas públicas da agricultura familiar local. $\mathrm{O}$ modelo mostrou que os únicos alimentos eficientes foram as Frutas e Legumes. Cereais e leguminosas, Laticínios, Farinha e produtos açucarados, Farinha, Amido e Massas, Produtos de panificação, Carnes processadas, Óleos e gorduras, Aves e ovos e outros não apresentaram eficiência [ver Anexo I].

Esse resultado mostra que, dentre os alimentos ofertados para as escolas públicas de Goiânia, os alimentos mais eficientes são aqueles considerados nutritivos e saudáveis. Portanto, aqueles que oferecem maior segurança alimentar para as crianças e jovens.

Isso mostra que a parceria entre agricultores familiares e escolas públicas, além de ser importante para o fortalecimento da agricultura familiar, dos pequenos agricultores e para o desenvolvimento da economia local, também contribui para o oferecimento de alimentos nutritivos. Mais importante, o oferecimento destes alimentos ocorre de forma eficiente, mais eficiente do que os alimentos processados.

No entanto, a baixa eficiência demonstrada pelo oferecimento de produtos industrializados ou semi-processados pode demonstrar uma desvantagem do modelo de negócio da agricultura familiar, o que pode ser investigado em estudos futuros. Isto porque, se a indústria alimentícia pode oferecer estes alimentos de forma mais eficiente, com preços mais competitivos, fazendo com que os pequenos agricultores percam espaço. Sendo assim, pesquisas futuras precisam analisar quais agentes são mais eficazes em oferecer os diferentes tipos de alimentos às escolas públicas, o que pode diferenciar os modelos de negócios a serem adotados.

\section{CONCLUSÃO}

Este artigo criou um mensurou a eficiência da oferta de alimentos, produzidos por pequenos agricultores, às escolas públicas. Os resultados mostraram que os alimentos saudáveis são os mais eficientes na parceria entre pequenos agricultores e escolas públicas (Economia Solidária), o que demonstra a importância deste modelo de negócio para a Segurança Alimentar.

Os alimentos processados não foram eficientes, o que mostra que a compra desses produtos precisa ser feita por meio de outros tipos de fornecedores (indústrias ou traders). Estudos futuros podem mapear quais produtos são adequados para cada tipo de fornecedor, levando em conta a logística e a estratégia de negócios.

Além disso, este artigo não analisou a eficiência dos próprios agricultores, da colheita, do uso da terra, ou as características observáveis dos envolvidos neste processo (idade, educação, entre outros), bem como as condições de trabalho. Estas são questões importantes para gerar eficiência, mas que podem ser exploradas por estudos futuros.

Finalmente, nossos resultados mostraram que a Economia Solidária e a Segurança Alimentar são importantes em dois aspectos. Primeiro, porque a 
parceria entre pequenos agricultores e escolas públicas desenvolve a economia local, beneficiando principalmente a agricultura familiar, geralmente caracterizada por trabalhadores de baixa renda. Em segundo lugar, a oferta de alimentos saudáveis e frescos produzidos localmente beneficia crianças e jovens nas escolas públicas por meio de uma dieta equilibrada e pode mudar hábitos alimentares no futuro.

\section{AGRADECIMENTOS}

O primeiro autor agradece à CAPES pelo financiamento do Programa 6492 - Doutorado CAPES/DAAD/CNPQ Processo no 88887.161388/2017-00. Os demais autores agradecem à FAPEG, ao CNPq e ao British Council pelo financiamento e por apoiarem esta pesquisa.

\section{REFERÊNCIAS}

[1] Hoffmann R. Brasil, 2013: mais segurança alimentar. Segur. Aliment. Nutr. 2014;21(2):422-436.

[2] Singer P. Introdução à Economia Solidária. Editora Fundação Perseu Abramo: São Paulo; 2002.

[3] Addor F. Desafio da Economia Solidária no Brasil: uma sistematização da literatura existente. SOLTEC/UFRJ: Rio de Janeiro; 2006.

[4] Coelho AB, Aguiar DRD, Fernandes EA. Padrão de consumo de alimentos no Brasil. Rev. Econ. Sociol. Rural. 2009;47(2):335-362.

[5] Rocha C. Developments in National Policies for Food and Nutrition Security in Brazil. Development Policy Review. 2009;27(1):51-66.

[6] Hoffmann R. Determinantes da Insegurança Alimentar no Brasil: Análise dos Dados da PNAD de 2004. Segur. Aliment. Nutr. 2008;15(1):49-61.

[7] Pizzani A, Rego WD. Vozes do Bolsa Familia: autonomia, dinheiro e cidadania. Editora Unesp: São Paulo; 2013.

[8] Hoffmann R. Elasticidades-renda das despesas e do consumo de alimentos no Brasil em 2002-2003. In: Silveira F et al. Gasto e consumo das famílias brasileiras contemporâneas. v. 2. Brasilia: IPEA; 2007. p. 463-484.
[9] Hoffmann R. Determinantes da insegurança alimentar no Brasil em 2004 e 2009. Segur. Aliment. Nutr. 2013;20(2):219235.

[10] Gaiger LIG. A Economia Solidária Diante do Modo de Produção Capitalista. Caderno CRH. 2003;39:181-211.

[11] Oliveira FCR. Alimentos normais, light/diet e orgânicos: o consumo segundo as classes econômicas e suas elasticidadesrenda [tese]. Piracicaba: Escola Superior de Agricultura "Luiz de Queiroz", 2014.

[12] Banerjee A, Duflo E, Goldberg N, Karlan D, Osei R, Pariente W, et al. A multifaceted program causes lasting progress for the very poor: Evidence from six countries. Science. 2015;348:6236.

[13] Mariano EB, Rebelatto DAD. Transformation of wealth produced into quality of life: analysis of the social efficiency of nation-states with the DEA's triple index approach. Journal of the Operational Research Society. 2014;65(11): 1664-1681.

[14] Charnes A, Cooper WW, Rhodes E. Measuring Efficiency of Decision-Making Units. European Journal of Operational Research. 1978;2(6):429-444.

[15] Cooper WW, Seiford LM, Tone K. Introduction to data envelopment analysis and its uses: with DEA-solver software and references. Springer Science \& Business Media: Berlim; 2006.

[16] Cook WD, Zhu J. Data Envelopment Analysis - A Handbook on the Modeling of Internal Structures and Networks. International Series in Operations Research \& Management Science. Springer Science \& Business Media: New York; 2014.

[17] Périco AE, Rebelatto DAN, Santana NB. Eficiência bancária: os maiores bancos são os mais eficientes? Uma análise por envoltória de dados. Gest. Prod. 2008;15(2):421-431.

[18] Périco AE, Santana NB, Rebelatto DAN. Eficiência dos aeroportos internacionais brasileiros: uma análise envoltória de dados com bootstrap. Gestão \& Produção. 2017;24:370-381.

[19] Camioto FDC, Mariano EB, Rebelatto DAD. Efficiency in Brazil's industrial sectors in terms of energy and sustainable development. Environmental Science \& Policy. 2014;37:50-60.

[20] Ferraz D, Moralles HF, Campoli JS, Oliveira FCR, Rebelatto DAN. Economic Complexity and Human Development: DEA performance measurement in Asia and Latin America. Gestão \& Produção. 2018;25(4):839-853. DOI https://dx.doi.org/10.1590/0104-530x3925-18 
[21] Mariano EB, Rebelatto DAD. Transformation of wealth produced into quality of life: analysis of the social efficiency of nation-states with the DEA's triple index approach. Journal of the Operational Research Society. 2014;65(11):1664-1681.

[22] Camioto FC, Rebelatto DAN, Rocha RT. Análise da eficiência energética nos países do BRICS: um estudo envolvendo a Análise por Envoltória de Dados. Gestão \& Produção. 2016;23:192-203.

[23] Dhungana BR, Nuthall PL, Nartea GV. Measuring the economic inefficiency of Nepalese rice farms using data envelopment analysis. Australian Journal of Agricultural and Resource Economics. 2004;48(2):347-369.
[24] Mariano EB, Sobreiro VA, Rebelatto DAD. Human development and data envelopment analysis: A structured literature review. Omega-International Journal of Management Science. 2015;54:33-49.

[25] Despotis D. A reassessment of the human development index via data envelopment analysis. Journal of the Operational Research Society. 2005;56(8):969-980.

[26] Despotis D. Measuring human development via data envelopment analysis: the case of Asia and the Pacific. Omega. 2005;33(5):385-390. 


\section{ANEXO I}

Tabela 2. Resultados do Cálculo de Eficiência

\begin{tabular}{|c|c|c|}
\hline Produtos & Tipo & Eficiência \\
\hline Couve flor & Vegetais e outros & 1.00 \\
\hline Jiló & Vegetais e outros & 1.00 \\
\hline Almeirão & Vegetais e outros & 1.00 \\
\hline Beterraba & Tubérculo & 1.00 \\
\hline Açafrão & Tubérculo & 1.00 \\
\hline Batata Doce & Tubérculo & 1.00 \\
\hline Pimenta verde & Vegetais e outros & 1.00 \\
\hline Hortelã & Vegetais e outros & 1.00 \\
\hline Inhame & Vegetais e outros & 1.00 \\
\hline Manjericão & Vegetais e outros & 1.00 \\
\hline Salsinha & Vegetais e outros & 1.00 \\
\hline Tomate & Frutas & 1.00 \\
\hline Melancia & Fruitas & 1.00 \\
\hline Cheiro Verde & Vegetais e outros & 0.97 \\
\hline Repolho & Vegetais e outros & 0.94 \\
\hline Milho Verde & Comida enlatada & 0.90 \\
\hline Alface & Vegetais e outros & 0.88 \\
\hline Chuchu & Vegetais e outros & 0.88 \\
\hline Mandioca & Farinha e Semolina & 0.85 \\
\hline Cebola & Vegetais e outros & 0.84 \\
\hline Repolho & Vegetais e outros & 0.84 \\
\hline Alho & Vegetais e outros & 0.83 \\
\hline Agrião & Vegetais e outros & 0.82 \\
\hline Batata & Tubérculo & 0.78 \\
\hline Mandioca descascada & Tubérculo & 0.77 \\
\hline Cenoura & Vegetais e outros & 0.77 \\
\hline Coentro & Vegetais e outros & 0.76 \\
\hline Vagem & Vegetais e outros & 0.70 \\
\hline Acelga & Vegetais e outros & 0.69 \\
\hline Cará & Vegetais e outros & 0.67 \\
\hline Laranja & Frutas & 0.66 \\
\hline Laranja Pera & Frutas & 0.63 \\
\hline Brócolis & Vegetais e outros & 0.63 \\
\hline Abacaxi & Frutas & 0.59 \\
\hline Limão & Frutas & 0.58 \\
\hline Manga & Frutas & 0.58 \\
\hline Couve-flor & Vegetais e outros & 0.53 \\
\hline Feijão & Cereais e legumes & 0.52 \\
\hline Queijo & Lacticínios & 0.52 \\
\hline Soja & Cereais e legumes & 0.51 \\
\hline Polpa de frutas & Açúcar e produtos de confeitaria & 0.50 \\
\hline Farinha de soja & Farinha e Semolina & 0.49 \\
\hline Abóbora & Vegetais e outros & 0.48 \\
\hline Abacate & Frutas & 0.47 \\
\hline Canjica de milho & Cereais e legumes & 0.46 \\
\hline Queijo ralado & Lacticínios & 0.44 \\
\hline Milho & Cereais e legumes & 0.43 \\
\hline
\end{tabular}




\begin{tabular}{|c|c|c|}
\hline Produtos & Tipo & Eficiência \\
\hline Mamão & Frutas & 0.43 \\
\hline Doces industrializados & Açúcar e produtos de confeitaria & 0.41 \\
\hline Banana & Frutas & 0.40 \\
\hline Leite pasteurizado tipo C & Lacticínios & 0.39 \\
\hline Rúcula & Vegetais e outros & 0.39 \\
\hline Cebolinha & Vegetais e outros & 0.38 \\
\hline Polpa de caju & Açúcar e produtos de confeitaria & 0.37 \\
\hline Polpa de maracujá & Açúcar e produtos de confeitaria & 0.37 \\
\hline Polpa de morango & Açúcar e produtos de confeitaria & 0.36 \\
\hline Tamarindo & Açúcar e produtos de confeitaria & 0.36 \\
\hline Quiabo & Vegetais e outros & 0.35 \\
\hline Pão de queijo & Pães & 0.32 \\
\hline Salsicha Suína & Carnes processadas & 0.30 \\
\hline Polpa de Abacaxi & Açúcar e produtos de confeitaria & 0.30 \\
\hline Pepino & Vegetais e outros & 0.26 \\
\hline Salsicha de frango & Carnes processadas & 0.26 \\
\hline Tangerina & Frutas & 0.25 \\
\hline Óleo de soja & Óleos e Gorduras & 0.25 \\
\hline Ovos & Aves e ovos & 0.25 \\
\hline Arroz & Cereais e legumes & 0.24 \\
\hline Tangerina tipo 2 & Frutas & 0.24 \\
\hline Bolos & Açúcar e produtos de confeitaria & 0.24 \\
\hline Proteína de Soja & Farinha e Semolina & 0.24 \\
\hline Rosca Doce & Pães & 0.22 \\
\hline Mexerica Ponkan & Frutas & 0.21 \\
\hline Melão & Frutas & 0.20 \\
\hline Queijo mussarela & Lacticínios & 0.19 \\
\hline Polpa de Cajá & Açúcar e produtos de confeitaria & 0.18 \\
\hline Polpa de Acerola & Açúcar e produtos de confeitaria & 0.18 \\
\hline Iogurte & Lacticínios & 0.18 \\
\hline Massa alimentícia de milho & Farinha e Semolina & 0.18 \\
\hline Rapadura & Açúcar e produtos de confeitaria & 0.17 \\
\hline Pão Caseiro & Pães & 0.13 \\
\hline Suco & Diversos & 0.12 \\
\hline Maçã & Frutas & 0.12 \\
\hline Bebida láctea & Lacticínios & 0.09 \\
\hline Doce de leite enlatado & Açúcar e produtos de confeitaria & 0.03 \\
\hline Rabanete & Tubérculo & 0.02 \\
\hline Polvilho Azedo & Pães & 0.02 \\
\hline Polvilho Doce & Pães & 0.02 \\
\hline Queijo Ralado Tipo 2 & Lacticínios & 0.02 \\
\hline Maracujá azedo & Frutas & 0.02 \\
\hline Manteiga & Lacticínios & 0.01 \\
\hline
\end{tabular}

